\title{
Tangence
}

\section{Solitude entre deux rives}

\section{Sergio Kokis}

Numéro 59, janvier 1999

Écrivains d'ailleurs

URI : https://id.erudit.org/iderudit/025998ar

DOI : https://doi.org/10.7202/025998ar

Aller au sommaire du numéro

Éditeur(s)

Tangence

ISSN

0226-9554 (imprimé)

1710-0305 (numérique)

Découvrir la revue

Citer cet article

Kokis, S. (1999). Solitude entre deux rives. Tangence, (59), 133-137.

https://doi.org/10.7202/025998ar d'utilisation que vous pouvez consulter en ligne.

https://apropos.erudit.org/fr/usagers/politique-dutilisation/ 


\section{Solitude entre deux rives}

\section{Sergio Kokis}

Platon n'accorde pas droit de cité au poète.

Blaise Cendrars, "Ma danse", 1914

Si l'on accepte que la vision originale, le changement de perspective et la modification des habitudes sont l'essence même de la connaissance créatrice, la métaphore du batelier passeur pourrait servir d'image pour aider à comprendre la place de l'artiste dans le monde. Je suis persuadé que cela s'applique aussi à l'homme de science mais je me limiterai ici à l'artiste de la parole, de la narration.

L'écrivain serait alors comme le batelier, celui qui s'est libéré de la matérialité immédiate, concrète, de l'utilitarisme de sa propre langue pour tenter de traduire (traducere ou übersetzen dans leurs acceptions originales) dans une langue publique les réalités d'une autre rive, d'une autre culture, d'un autre imaginaire. Comme le passeur, il est suspendu entre deux mondes, dans une position extrêmement solitaire puisqu'elle rompt justement à la fois avec la réalité intime et avec la quotidienneté pratique des actions humaines. L'être de celui qui se déplace est dans le chemin, entre deux places, et il se revendique comme un déplacé.

Mieux que d'autres, Sartre a su exprimer cette position libre et instable de l'homme de lettres lorsqu'il la définit en faisant appel au concept de bâtardise. Le bâtard est justement "l'enfant conçu dans la grange" (bantsi), entre deux conditions sociales, sans jamais pouvoir appartenir de droit au rang supérieur ni se conformer au rang inférieur. Déshérité de ses droits mais les évoquant dans son désir, celui-ci est condamné à fréquenter des mondes opposés par l'imaginaire et dans l'imaginaire, dans un rôle (masque, persona) d'éternel exilé. Si sa situation sociale peut paraître inconfortable, ses perspectives au contraire sont multiples, sa marge de liberté est plus vaste et son sentiment d'individualité est bien plus aigu. Son véritable lieu est le flux du courant 
134

de la vie et, tout en possédant les idiomes des deux rives, il devra se débattre sans cesse avec une langue étrangère, originale, une langue apprise et inventée qu'il se devra de conquérir chaque jour pour réussir ses propres actes de parole. C'est d'ailleurs plus qu'une boutade, l'affirmation selon laquelle l'écrivain écrit toujours dans une langue étrangère. De même, l'acte de communication quel qu'il soit implique nécessairement l'aliénation de soi dans une traduction puisque son but est d'atteindre l'autre. L'acte d'amour, toute sensualité le confirment: on doit créer l'autre dans sa propre langue mais à notre image, tout en le laissant libre de nous créer à son tour dans notre langue personnelle. Sinon, il n'y a pas de transmission de sens.

Le concept d'art en général se conforme toujours à sa racine indo-européenne (are) qui signifie ajuster, adapter; celle-ci évolue dans le grec pour donner naissance aux concepts d'articuler (arthros), de juste rapport (barmonia), de nombre (arithmos). En latin elle produit ritus (correction des pratiques magiques et religieuses) et arte (métier, savoir-faire). L'idée de médiation y est prédominante. Par ailleurs, aux origines du concept de création nous retrouvons la racine $k(e) r e ́$, qui a le sens de semence, de transition entre deux moments de vie. La même idée de passage, d'intermédiaire, existe dans le concept allemand de schöpfen (scephen), qui veut dire créer mais aussi puiser, sortir l'eau d'un puits, la faire traverser un espace vertical d'un lieu à un autre, et ainsi la changer de point de vue et de sens selon l'agent observateur.

Il n'est donc pas étonnant que dans leur réalité existentielle, le bâtard, le rêveur, le barbare (barbaros: qui ne parle pas le grec) ou le déraciné aient depuis toujours personnifié le changement, la poésie (poiein : acte de faire du nouveau). Les thèmes du voyage et de l'exil sont d'ailleurs étroitement liés à l'art, comme le suggère l'image de l'aède Homère, aveugle aux choses concrètes et voyageant de ville en ville pour chanter l'homme face au mythe. Cette figure devient d'autant plus frappante dans notre siècle caractérisé par la transhumance. En effet, autrefois l'artiste des métropoles s'exprimait la plupart du temps à l'intérieur d'une même culture. Sa bâtardise venait non pas de sa situation factuelle mais bien de son attitude critique, qui le mettait nécessairement à l'extérieur de son objet de narration. Balzac, par exemple, comme observateur et analyste, se situe en dehors du monde bourgeois qu'il décrit si bien. Cervantes est aussi un exemple frappant de cette extériorité : seule une méta-position à la fois 
extérieure, intérieure et synthétique lui donne accès avec tant d'argutie à l'univers littéraire de l'Espagne de son temps et à l'art éternel de la narration humaine. C'étaient, comme tant d'autres, des immigrés de l'intérieur, des exilés spirituels; ce n'est pas étonnant que beaucoup d'entre eux aient été aussi des étrangers ou des bannis au sens propre du terme.

Avec la rupture des cloisons entre les métropoles et le monde des colonies, notre siècle a connu une radicalisation substantielle du facteur déracinement dans l'extension du concept d'artiste. D'abord en Europe, à la suite des conflits politiques et armés depuis avant même la Première Guerre mondiale. Je pense à Conrad, à Tzara, à Romain Rolland et à Barbusse entre autres, sans compter les exodes et les divers exils produits par le nazisme et par le communisme. La tendance s'est ensuite accentuée avec l'afflux formidable des étrangers venus des contrées périphériques et qui ne cessent d'envahir les capitales du nord. Ces nouveaux barbares - à la façon des militaires germaniques qui occupaient peu à peu les armées romaines en tant que soldats, puis officiers, pour ensuite devenir des politiciens au sein du pouvoir - possèdent de mieux en mieux la langue des colonisateurs, laquelle est la seule qui leur donne accès à l'universalisation de leur imaginaire d'origine. Ils immigrent, ils s'installent et, au fur et à mesure, ils introduisent la façon d'être de leurs mondes respectifs, leurs histoires et leur sensualité dans la structure cognitive et linguistique des métropoles. Surtout, ils réveillent les thèmes de la rupture des rapports coloniaux et des luttes sociales. Davantage que la nostalgie, le thème constant de ces nouvelles littératures est le monde éclaté et la rupture des mythes tels que le vivent de façon quotidienne ces écrivains venus d'ailleurs.

Le choix de la langue étrangère est une évidence, j'insiste; c'était aussi évident que le barbare devait d'abord accepter le cadre des disciplines militaires romaines pour se faire accepter dans leurs légions. Sauf que ce choix n'est pas inerte. La langue, sa syntaxe et son lexique sont intimement liés à l'imaginaire de celui qui parle, à sa conception du monde. En particulier, la prosodie de l'étranger se glisse de façon subversive dans la narration, apparemment sans faire violence à la syntaxe. Cette nouvelle mélodie minera les habitudes au sein même de la conscience du lecteur métropolitain. Ce phénomène a déjà été étudié à propos de la décadence de l'empire romain et de la Renaissance. Plus près de nous, l'impact des influences africaines et autochtones a 
136

été décisif pour la formation des idiomes et de l'imaginaire du continent américain. Le thème mexicain du métissage, dont la figure de la Malinche est le paradigme politique mais surtout linguistique, obéit à une dynamique semblable. En outre, on parle de l'essor actuel des paroles créoles mais on tend à oublier que "créole", dans son origine portugaise, veut dire serf élevé dans la Casa grande des maîtres plutôt que dans la senzala des esclaves (criado): donc, bâtard et s'exprimant dans une langue seconde. Le terme garde d'ailleurs son acception de mulâtre dans le portugais brésilien. Mais, roman aussi, ne voulait-il pas dire un récit en langue vulgaire (romanz) par opposition aux récits en langue latine?

Lorsqu'on médite un tant soit peu sur ces quelques considérations à peine esquissées, il me paraît clair que l'usage du terme de littérature allophone (faite par des écrivains venus d'ailleurs) est une erreur de jugement. Il s'agit d'une notion fallacieuse qui tend à suggérer qu'il puisse y avoir une littérature spécifiquement autochtone en dehors des sociétés mythiques, religieuses, closes. Conrad serait-il un auteur de littérature allophone? Et que dire de B. Traven, d'Elsa Triolet, de Nabokov ou de José Maria Arguedas parmi tant d'autres?

Il est vrai, cependant, que dans des sociétés fermées, totalitaires, on a tenté de produire une littérature non critique, assujettie au pouvoir, et dont les thèmes avaient trait à la race, au sang, à la famille, à la religion, aux traditions, à la pureté de la langue et des mœurs, lorsque ce n'était ouvertement la haine des étrangers ou des déviants. On se souviendra avec profit de comment Platon (République, livre II) propose d'émasculer les textes d'Hésiode et d'Homère entre autres, pour le bien de sa société totalitaire. Hélas! les exemples de ce type existent toujours en notre fin de siècle, où nous nous débattons avec la plaie des historicismes de tout acabit ou des intégrismes religieux et nationalistes. Cela n'est pas de l'art mais de la simple propagande. Les artistes répudient ces relents funestes et ils persistent dans l'expression de leurs imaginaires multiples en se tournant vers des avenirs ouverts. Les lecteurs les suivent dans cette voie de liberté car l'exercice de l'imagination est un acte de décentration et de rupture, même lorsqu'il est effectué sous la forme de simple lecture d'un texte. Rêver (vagus) n'est-il pas aussi à l'origine de errer (vagabundus), vaguer, divaguer - c'est-à-dire rompre avec les choses telles qu'elles sont, pour les concevoir telles qu'elles pourraient être? 
Je crois que cette tendance qui cherche à diviser les littératures en allophones et autochtones n'est que le sous-produit d'idéologies passéistes et franchement rétrogrades. De toute façon, les artistes et les barbares sont là pour rester. Et tant qu'il y aura un germe de liberté dans la solitude des êtres humains, le saltimbanque, l'aède errant, le conteur et le magicien charmeront les esprits. Seuls les larbins des liturgies clameront qu'il s'agit de dangereux étrangers ou de fauteurs de troubles. 